\title{
Comparative Studies of Breast Cancer Risk Prediction Models as Screening Tools for Women in Low -and Middle-income Societies
}

Ceasar Dubor Danladi

Near East University

Nedime Serakinci ( $\square$ nedimeserakinci@gmail.com )

Near East Univeristy

\section{Research Article}

Keywords: Breast cancer, Mammogram, Risk prediction models, Low -and Middle-income societies

Posted Date: February 25th, 2022

DOI: https://doi.org/10.21203/rs.3.rs-1395809/v1

License: (c) (1) This work is licensed under a Creative Commons Attribution 4.0 International License.

Read Full License 


\section{Abstract \\ Background}

Early detection of breast cancer alters the prognosis and tools that can predict the risk for breast cancer in women will have a significant impact on health care systems in low -and middle-income regions.

\section{Objective}

We compared the performances of the Breast and Ovarian Analysis of Disease Incidence and Carrier Estimation Algorithm (BOADICEA) model, International Breast Cancer Intervention Study (IBIS) model and Gail model in predicting the risk of breast cancer in the women of North Cyprus.

\section{Methods}

655 women were enrolled in the study consisting of 318 breast cancer cases and 337 hospital-based controls. Data were obtained from medical records and one-on-one interviews after informed consent.

\section{Results}

From the receiver operating curves $(\mathrm{ROC})$ the models AUCs were $0.81(95 \% \mathrm{C} . \mathrm{I}=0.77-0.84)$ for BOADICEA, IBIS 0.80(95\%C.I $=0.77-0.84)$ and Gail $0.76(95 \%$ C.I $=0.73-0.80)$ respectively.

\section{Conclusions}

The studied breast cancer risk prediction models are suitable, simple, cost-effective, and non-invasive tools for identifying high-risk women from low- and middle-income regions that can benefit from mammography screening. There were similar performances amongst the models, at a closer look the BOADICEA and IBIS models performed slightly better.

\section{Introduction}

Breast cancer (BC) is a disease that has remained the leading cause of death in women worldwide (1). In 2018, more than 2 million women were diagnosed with BC in 140 of 184 nations (2). BC incidence and deaths occur mostly in low -and middle-income countries (3). One-half of all BC cases exist in low -and middle-income societies, with $62 \%$ of the world's BC deaths. (4) Whereas, in the United States approximately 249,260 new cases of BC are diagnosed each year, and the mortality rate is decreasing, (5) with $89.7 \%$ 5year survival rate. (6) This portrays an inequality in the global health standards. Globally the 
incidence of BC varies from 19.3 per 100,000 women in Eastern Africa to 89.7 per 100,000 in Western Europe, With incidence below 40 per 100,000 in majority of developing countries. (7)

Northern Cyprus, can be regarded as a middle-income society, small, enclosed, ideal for epidemiological research, has a typical western Mediterranean lifestyle with living conditions and diets that should be favorable for good health (8) Previously, Hincal et al. investigated the prevalence of cancer in Northern Cyprus compared to different European countries between 1990-2004, and showed that breast cancer was the most common cancer in women and diagnosed at a lower average age than Northern and Southern Europe (9). Later on, Pervaiz et al. also found that out of 1395 enlisted cancer cases between 2007-2012, 665(47.67\%) were women, and BC was the most common cancer type among women (10).

Despite the fact mammography screening has been supported for the early detection of $\mathrm{BC}$, because of the large population of women, underfunding, infrastructure and human expertise needed, it is not costeffective and feasible for developing countries $(12,13)$.

Therefore, such countries can benefit from cost-effective and efficient risk assessment screening strategies that do not depend on tertiary or specialized health care. Denny et al explored some costeffective methods that can be used to reduce the gap between developing countries and developed countries for BC early detection, prevention, and care. (14) Risk prediction models can also serve as an additional simple, cost-effective, and supportive non-invasive method for identifying high-risk women that can benefit from mammography and preventive treatments.

Currently, several comprehensive BC risk assessment tools exist that incorporate various risk factors for the calculation of BC risk (1). Demographic and biomedical information such as age, reproductive factors (low parity, late age at first pregnancy, not breastfeeding, early menarche, late menopause) linked to longterm endogenous and exogenous hormone exposure, Body Mass Index (BMI), Family history and BRCA 1 and 2 mutation (15) and breast density (16) were used as input variables in the risk assessment models. $\mathrm{BC}$ risk can only be affected modestly by each of this risk factors but the risk level can be modified significantly in combination with family history and genetic factors. (11)

BC risk assessment models are empirical or genetic (17) such as, The Breast and Ovarian Analysis of Disease Incidence and Carrier Estimation Algorithm (BOADICEA) model (18), the International Breast Cancer Intervention Study (IBIS) model (19) and the National Cancer Institute's Breast Cancer Risk Assessment Tool (BCRAT) also known as the "Gail Model"(20). Though several risk assessment models have been proposed, the selection of these models for this study was based on their performances (17) and their discriminatory accuracies. (17) Their known performances in predicting high-risk women reassure us that these models will be able to aid in streamlining candidates for mammography and early identification of high-risk women will lead to early preventive interventions (21) that will save lives. Predictions derived from the models are more frequently used in the development of guidelines and recommendations for clinical care, in which women with a higher predicted risk are advised to initiate earlier mammography screening and to consider screening, genetic testing and chemoprevention. 
Therefore in this study, we compared the performances of the BOADICEA, IBIS, and Gail models in predicting the risk of breast cancer in women of North Cyprus.

\section{Materials And Methods}

This study was carried out in the hospital, Dr. Burhan Nalbantoglu Devlet Hastanesi, Lefkosa, North Cyprus. This hospital treats all BC cases in North Cyprus. Ethical approvals were obtained from the Near East University scientific research evaluation ethics community and Dr. Burhan Nalbantoglu Devlet Hastanesi's ethics community before the research was carried out. All methods were performed following the relevant guidelines and regulations.

\section{Sampling size was based on the following calculations:}

$$
n=\frac{N^{*} t^{2} p^{*} q}{(N-1) d^{2}+t^{2} * p^{*} q}
$$

\section{Equation 1}

Equation 1 was used to determine how many women are needed in order to get results that reflect the target population as precisely as possible. Were, $\mathbf{N}=121257$ (Women Population Size) This is the total number of women in the whole population, $t=t$-table value $=1.96(a=0.05) t$ is the number of standard deviations a given proportion is away from the mean, $p=$ (prevalence rate) $=91 / 100000=$ $\mathbf{0 . 0 0 0 9 1}$ (Expected Frequency) This is the proportion of the population affected with $B C, \mathbf{q}=1-p$ $=0.99909$ This is the proportion of the population not affected with BC, $\mathbf{d}=$ (Acceptable margin of Error) $=0,001$ The margin of error is the amount of error that can be tolerated. Lower margin of error requires a larger sample size. Following the calculations the required sample size $=317.8$ women.

\section{Study group:}

This was a study consisting of 655 women that were separated into two groups as follows: Case group = 318 women with confirmed cases of $\mathrm{BC}$ were included. The patients with $\mathrm{BC}$ were registered with the center's database and diagnosed based on pathological report according to the international classification of diseases for oncology 3rd edition (C50.0 - C50.9)(22). Hospital-based control groups $=$ 337 women without BC. Women with history of lobular or ductal carcinoma in-situ were excluded from the controls. Only participants between the ages of 30 to 84 years were included in the study group. Informed consent to participate was obtained after the aim of the study was explained by a medical professional.

\section{Data collection:}


Retrospective medical and demographic information of case group and hospital-based control group was collected from medical records and the information not available in the medical records was collected through one-on-one interviews.

A retrospective data was used because of the long latency period to $B C$ manifestation and the dynamic nature of the population, thus making it difficult for follow-up.

The Information collected included: age, age at diagnosis, age at menarche, age at first delivery, menopausal status, presence or absence of benign breast disease, history of $B C$ in first-degree relatives (FDR) or other relatives, BRCA 1 and 2 mutation, BMI, history of hormone replacement therapy (HRT) including estrogen/progestin and breast density.

\section{Breast cancer risk assessment:}

The information collected was used in the models to predict the risk of BC. The information on BRCA 1 and 2 mutations status could not be provided by the participants so it was excluded.

The IBIS model is a computer-based program that provided a woman's overall risk of BC by incorporating genetic determinants such as the BRCA 1 and 2 genes (19), Details about breast/ovarian cancer among family members, personal risk factors such as age, BMI, age at menarche, parity, age at first child, menopausal status, breast density, age at menopause, and benign breast disease (19). The IBIS model accommodates residual familial correlation by incorporating a latent common autosomal dominant lowrisk gene (23). The IBIS or Tyrer-Cuzick BC risk evaluation tool version $8.0 \mathrm{~b}$ used is available at (http://www.ems-trials.org/riskevaluator/). The performance of the IBIS model was measured by estimating the $\mathrm{BC}$ risk for each individual. The 10year risk was divided by 2 to obtain the 5year risk. Though $B C$ risk increases with age dividing the 10year risk gave an approximate value for the 5year risk. The BOADICEA model calculated 5year risk of BC in the women based on their age, family history, BRCA 1 and 2 carrier probabilities and includes a polygenic component, which allows for the familial correlation that is not captured by mutations in BRCA1 or 2. The BOADICEA risk calculation was carried out using BWAv3 (http://ccge.medschl.cam.ac.uk/boadicea/). The National Cancer Institute's online version of the breast cancer risk assessment tool (BCRAT) also known as the Gail model available at (http://www.cancer.gov/bcrisktool/) was also used and has questions about the 5 year BC risk based on age, age at menarche, age at first life birth, FDR with breast cancer, previous breast biopsies with or without atypical hyperplasia, BRCA mutation and race. White race/ethnicity (Caucasians) variables was used for all the women in this study in estimating their risks. For the Gail model five-year risk assessment, a rate of less than $1.67 \%$ was defined as low risk while a rate of $1.67 \%$ or more was defined as high risk (23). Based on the NCCN (2016) recommendation for prophylactic treatment for women the cut of value of $1.67 \%$ for $5 y e a r$ risk was used for all they models while categorizing high and low risk women (33).

\section{Statistical analysis:}


The Receiver Operating Characteristic Curve (ROC) plots, was utilized to measure the model's discriminative capacities. The c-statistics ranges from 0.5 (No discriminative ability) to 1.0 (Perfect discrimination). This determines whether the models will yield a higher risk for $\mathrm{BC}$ cases and lower risk for hospital-based controls. The predicted scores were used to distinguish between high and low risk. Sensitivities and specificities of the models were estimated for 5year BC risk at $1.67 \%$. The sensitivity test has the diagnostic ability to detect true positives and the specificity, the diagnostic ability to detect true negatives. A cut off value of $1.67 \%$ was used to predict the risk of BC.

The predictive accuracies of correctness (PAC) and the Nagelkerke's R square of the models were analyzed by logistic regression of the scores predicted by the models. The PAC measures how well the models fit the samples. The Nagelkerke's $R$ square is an adjusted version of the Cox and Snell $\mathrm{R}$ square that adjust the scale of the statistic to cover the full range from 0 to 1 . A perfect model has a theoretical maximum value of less than 1.All statistical analysis was done using SPSS version 24.0 analytical software.

\section{Results}

655 women were in the study group consisting of $318 \mathrm{BC}$ cases and 337 hospital-based controls. Table 1 summarizes the baseline characteristics of the participants. 
Table 1

The prevalence of risk factors in the study population

\begin{tabular}{|c|c|c|c|}
\hline Characteristics & Breast cancer patients & Population based controls & Total \\
\hline \multicolumn{4}{|l|}{ Age: } \\
\hline $30-39$ years & 6 & 128 & 134 \\
\hline $40-49$ years & 44 & 74 & 118 \\
\hline $50-59$ years & 56 & 57 & 113 \\
\hline $60-69$ years & 97 & 52 & 149 \\
\hline $70-85$ years & 115 & 26 & 141 \\
\hline \multicolumn{4}{|l|}{ Age at first birth: } \\
\hline$<20$ years & 56 & 47 & 103 \\
\hline $20-24$ years & 128 & 95 & 223 \\
\hline $25-29$ years & 75 & 67 & 142 \\
\hline$>=30$ years & 34 & 9 & 43 \\
\hline Nulliparous & 25 & 119 & 144 \\
\hline \multicolumn{4}{|l|}{ Age at menarche: } \\
\hline$<12$ years & 18 & 3 & 21 \\
\hline $12-13$ years & 217 & 252 & 469 \\
\hline$>=14$ years & 83 & 82 & 165 \\
\hline \multicolumn{4}{|l|}{ Breast biopsy: } \\
\hline Yes & 81 & 33 & 114 \\
\hline No & 237 & 304 & 541 \\
\hline \multicolumn{4}{|l|}{ Breast density: } \\
\hline Extremely dense & 26 & 38 & 64 \\
\hline Heterogenously dense & 187 & 144 & 331 \\
\hline Almost entirely fatty & 105 & 155 & 260 \\
\hline \multicolumn{4}{|l|}{ Breast feeding duration: } \\
\hline$>=24$ months & 165 & 87 & 252 \\
\hline $12-18$ months & 99 & 108 & 207 \\
\hline$<=6$ months & 29 & 19 & 48 \\
\hline
\end{tabular}




\begin{tabular}{|llll|}
\hline Characteristics & Breast cancer patients & Population based controls & Total \\
\hline Never & 25 & 123 & 148 \\
\hline FDR with BC: & 49 & 16 & 65 \\
\hline Yes & 269 & 321 & 590 \\
\hline No & & & 41 \\
\hline Second degree relatives with BC: & 35 & 6 & 614 \\
\hline Yes & 283 & 331 & 15 \\
\hline No & & & 640 \\
\hline HRT: & 15 & 0 & \\
\hline Yes & 303 & 337 & 363 \\
\hline No & & & 13 \\
\hline Menopausal & & 250 & 279 \\
\hline Status: & 113 & 34 & \\
\hline Premenopausal & 10 & & \\
\hline Perimenopausal & 195 & & \\
\hline Postmenopausal & & & \\
\hline
\end{tabular}

\section{Risk prediction models analysis:}

The models performances were evaluated as overall good. Using logistic regression analysis of the predicted scores, the BOADICEA, Gail and IBIS models displayed Nagelkerke R square of 0.32, 0.19 and 0.23 respectively. The Predictive accuracy of correctness (PAC) of the models is BOADICEA $72.4 \%$, IBIS $71.1 \%$, and Gail $69.9 \%$ respectively.

The discriminatory capacities of the models were obtained from the ROC curve and the Area Under the Curves (AUCs) as shown in (Table 3 ) are as follows: BOADICEA model AUC $=0.81(95 \% \mathrm{C} . \mathrm{I}=0.77-0.84$ ), IBIS model AUC $=0.80(95 \% \mathrm{C} . \mathrm{I}=0.77-0.84)$ and Gail model AUC $=0.76(95 \% \mathrm{C} . \mathrm{I}=0.73-0.80)$.

At a cut-off point of about $1.67 \%$, the sensitivities of the model's in predicting a high-risk woman among the breast cancer cases were as follows: $\mathrm{BOADICEA}=26.41 \%$, IBIS $=19.4 \%$, and Gail $=17.3 \%$. (Table 2 ) 
Table 2

The models sensitivities and specificities at different cut-off points

\begin{tabular}{|lll|}
\hline Models & Sensitivity & Specificity \\
\hline Cut-off $\sim 1.67 \%$ & $(19.4 \%)$ & $(97.3 \%)$ \\
IBIS & $(26.41 \%)$ & $(98.8 \%)$ \\
BOADICEA & $(17.3 \%)$ & $(98.5 \%)$ \\
Gail & & \\
\hline
\end{tabular}

Table 3

Table for area under the curve for the risk prediction models

\begin{tabular}{|llllll|}
\hline Models & Area & S.E & Sig. & \multicolumn{2}{c|}{$95 \%$ C.I } \\
\cline { 5 - 6 } & & & & Lower & Upper \\
\hline BOADICEA Model & 0.81 & 0.17 & $<0.001$ & 0.77 & 0.84 \\
\hline IBIS Model & 0.80 & 0.17 & $<0.001$ & 0.77 & 0.84 \\
\hline Gail Model & 0.76 & 0.19 & $<0.001$ & 0.73 & 0.80 \\
\hline
\end{tabular}

\section{Declarations}

We found that all they models performed good in predicting the risk of $B C$ in women. Demographic and biomedical data of the participants were used to predict the risk of $\mathrm{BC}$ by the models. Our results demonstrated that the Gail, IBIS and BOADICEA models are suitable for predicting BC. Though The BOADICEA and IBIS model gave a slightly better predictive value, all the models showed a reasonable predictive accuracy. It is important for a risk prediction model to have a good predictive accuracy (24). The estimated risk of $\mathrm{BC}$ varied between the risk models because each model uses different statistical calculation for the same variables and also different risk factors were used by each model for statistical calculation. The models used genetic factors such as family history in combination with other risk factors to enhance their stratification of BC risk. Inherited factors elucidate just about a quarter of BC risk (25). Meta and pooled studies have demonstrated that $\mathrm{BC}$ risk is around twice higher in women with one FDR with breast cancer, than women with no FDR. The risk increases with a larger number of affected FDR or relatives affected under 50 years (26). BRCA 1 and 2 mutations explain the molecular pathogenesis behind $15-20 \%$ of cases with first-degree family history $(27,25)$. While the remaining $80-$ $85 \%$ can be a result of breast density, age, menopausal status, history of HRT and age at menarche. Breast density seems heritable (28). But the mechanism underlying the association between breast density and $\mathrm{BC}$ is not yet understood. Though age and menopausal status impacts breast density, younger and premenopausal women in general have denser breast (29). Women with early age of menarche have a slight increase in $\mathrm{BC}$ risk. A woman with an early age at menarche will have an increase 
in the time of exposure to estrogens, thus increasing BC risk (30). HRT use is common among postmenopausal women and is linked to increased $\mathrm{BC}$ risk (31). In comparison to a recent validation study (35) the sensitivities of the models at $1.67 \%$ was low but the specificities were similar. The sensitivities can be improved by inputting information on genetic mutations such as BRCA 1 and 2 , which could not be provided by the participants.

Despite the rise in $\mathrm{BC}$ incidence in the women of North Cyprus there is no studies on risk models effectiveness on BC screening.

These models can serve as suitable simple non-invasive alternative screening for the identification of high-risk women, thus streamlining the focus of the limited mammogram resources to the right group in low-income and middle-income countries. Using these models will also reduce unnecessary mammography need and radiation exposure on potentially low-risk women. The use of the risk prediction models has additional advantages as it is not dependent on physical examination, easy to utilize and implement, being cost-effective and seems to enhance outcome and survival.

Risk prediction models can serve as additional screening tools that will yield effective results by identifying women at high-risk that will need immediate mammography, thus reducing the burden on the already constrained facilities and hence saving lives.

The risk assessment models can be incorporated into the mHealth features similar to the WHO initiative in 2012 thus empowering women. (32) Low-risk women through this can benefit from primary care.

While the models intend to ascertain the risk for an individual, the risk factors utilized depend on population risk from epidemiological investigations. Therefore, more studies have to be carried out among various populations of women in other to identify new lifestyle/environmental factors, biomarkers, genetic markers and incidence rates that are peculiar to that group, which can be incorporated into prospective risk models because the possibility of identifying those at high-risk would be enhanced by using a comprehensive risk model that integrates all known risk factors.

The weakness of our study is the fact that it was based on retrospectively collected data. However, the collecting process was done independently, so unlikely to have altered the results and caused bias. The AUC estimates are bound to be biased, Since our study was carried out on a case-control group, but this was minimized (34).

BRCA 1 and 2 information were not used because it is not a common test in the study setting and could not be provided by the participants, so a comprehensive family history of breast cancer, which explains the BRCA 1 and 2 mutation associations with about $20 \%$ breast cancer cases, was used alongside other risk factors $(27,25)$. Information on environmental risk factors was also not collected, because they were not considered risk predictors in the models and this may have created a gap in the awareness of interethnic risk factors in the studied population. 
Despite these biases and limitations the urgent need for these risk prediction models in providing relevant breast cancer control in developing societies outweighs the shortfalls.

\section{Conclusion}

Our results suggest that $\mathrm{BC}$ risk prediction models can be suitable, simple, cost-effective, and noninvasive tools for the identification of high-risk women in low-income and middle-income societies. It may serve as a gatekeeper for mammography and a radiation saving tool for low-risk women by reducing unnecessary mammography and thereby decreasing health costs. Risk prediction models can also be used in screening women left out of mammography due to limited facilities. Hence, these models need to be explored in developing regions where access to early detection, cancer care, and mammography is limited. Though all the model's performances were similar, at a closer look the BOADICEA and IBIS models were slightly better.

\section{Declarations}

\section{Acknowledgement:}

Our gratitude goes to the participants, for their time and willingness to respond to the interviews. Though names are numerous to be mentioned, we sincerely show our appreciation to the management and staff of the Cancer Center of Dr. Burhan Nalbantoglu Devlet Hastanesi, Lefkosa, North Cyprus, for their support and allowing us access their database.

Contributions of Authors: Ceasar Dubor Danladi designed the work, collected, analyzed and interpreted the data and also drafted the manuscript.

Prof.Dr.Nedime Serakinci designed the work, analyzed and interpreted the data and revised the manuscript.

Conflict of interest statement: There is no conflict of interest

\section{References}

1. Parkin DM, Bray F, Ferlay J, Pisani P. Global cancer statistics, 2002. CA Cancer J Clin 2005:55:74 108.

2. Bray, F.; Ferlay, J.; Soerjomataram, I.; Siegel, R.L.; Torre, L.A.; Jemal, A. Global Cancer Statistics 2018: GLOBOCAN estimates of incidence and mortality worldwide for 36 cancers in 185 countries. CA Cancer. J. Clin. 2018, 68, 394-424. [CrossRef] [PubMed]

3. de Sanjose S, Serrano B, Castellsague X, et al. Human papillomavirus (HPV) and related cancers in the Global Alliance for Vaccines and Immunization (GAVI) countries. A WHO/ICO HPV Information Centre Report. Vaccine 2012; 30 (suppl 4): D1-83, vi. 
4. Torre LA, Bray F, Siegel RL, Ferlay J, Lortet-Tieulent J, Jemal A. Global cancer statistics, 2012. CA Cancer J Clin. 2015;65((2)):87-108. doi: 10.3322/caac.21262.

5. Siegel RL, Miller KD, Jemal A. Cancer statistics, 2016. CA Cancer J Clin. 2016;66((1)):7-30. Doi: $10.3322 /$ caac. 21332 .

6. Allemani C, Weir HK, Carreira H, Harewood R, Spika D, Wang XS, et al. Global surveillance of cancer survival 1995-2009: analysis of individual data for 25,676,887 patients from 279 population-based registries in 67 countries (CONCORD-2) Lancet. 2015;385((9972)):977-1010. doi: 10.1016/S01406736(14)62038-9.

7. WHO (2008). The global burden of disease:2004 update.

8. Martinez-Gonzales M, Sanchez-Villegas, A. The emerging role of Mediterranean diets in cardiovascular epidemiology: Monosaturated fats, olive oil, red wine or the whole pattern? Eur J Epidemiol 2004:19, 9-13.

9. Hincal E, Taneri U, Djamgoz MB. Cancer incidence in North Cyprus(1994-2004) relative to European rates. Asian Pac J Cancer Prev 2008: 9:725-32.

10. Pervaiz R,Tulay P,Faisal F,Serakinci N. Incidence of Cancer in the Turkish republic of northern Cyprus. Turk J Med Sci. 2017:47(2):523-530.

11. Garcia-Closas M, Gunsoy NB, Chatterjee N. Combined associations of genetic and environmental risk factors: implications for prevention of breast cancer. J Natl Cancer Inst. 2014;106:dju305.

12. Sankaranarayanan R, Ramadas K, Qiao YL. Managing the changing burden of cancer in Asia. BMC Med 2014; 12: 3.

13. Lauby-Secretan B, Scoccianti C, Loomis D, et al. Breast-cancer screening-viewpoint of the IARC Working Group. N Engl J Med 2015; 372: 2353-58.

14. 10.1016/s0140-6736(16)31795-0.

15. Brandt A,Bermejo J,Sundquist J,Hemminki K. Age of onset in familial breast cancer as background data for medical surveillance.Br J Cancer 2010:102:42-7.

16. McCormack VA and dos Santos Silva I. Breast Density and parenchymal patterns as markers of breast cancer risk: a meta-analysis. Cancer Epidemiol Biomarkers Prev 2006:15: 1159-1169.

17. Amir E, Freedman OC, Seruga B, Evans DG. Assessing women at high risk of breast cancer: a review of risk assessment models. J Natl Cancer Inst 2010:102(10):680-691.

18. Antoniou AC, Cunningham AP, Peto J, et al. The BOADICEA model of genetic susceptibility to breast and ovarian cancers: updates and extensions. Br J Cancer 2008:98(8):1457-1466.

19. Tyrer J, Duffy SW, Cuzick J.A breast cancer prediction model incorporating familial and personal risk factors. Stat Med. 2004:23(7):1111-1130.

20. Costantino JP, Gail MH, Pee D, et al. Validation studies for models pro- jecting the risk of invasive and total breast cancer incidence. J Natl Cancer Inst. 1999:91(18):1541-1548.

21. Pickle LM, Johnson KA.Estimating the long-term probability of developing breast cancer. J Natl Cancer Inst 1989:81:1854-5. 
22. Sabatino SA,Burna Rb,Davis RB,Philips RS, Chen YH,McCarthy EP.Breast carcinoma screening and risk perception among women at increase risk for breast carcinoma: results from a national survey. Cancer 2004.100:2338-46.

23. Bevers TB,Anderson BO,Bonaccio E,et al. NCCN Clinical practice guidelines in oncology:breast cancer screening and diagnosis. J Natl compr Canc Netw 2009:1060-96.

24. Prascandola M. Ethics and breast cancer risk assessment. Ann Epidemiol 2000:10:461.

25. Lichtenstein P, Holm NV, Verkasalo PK, et al. Environmental and Heritable Factors in the Causation of Cancer - Analyses of Cohorts of Twins from Sweden, Denmark, and Finland. New Engl J Med 2000:343(2):78-85.

26. Barnard M, Boeke $C$, Tamimi R. Established breast cancer risk factors and risk of intrinsic tumor subtypes. Biochem Biophys Acta Rev Cancer 2015:1856:73-85.

27. Turnbull C, Rahman N.Genetic Predisposition to Breast Cancer: Past, Present, and Future. Ann Rev Genom Hum Genet 2008:9(1):321-45.

28. Martin LJ,Boyd NF. Mammographic density potential mechanisms of breast cancer risk associated with mammographic density: Hypotheses based on epidemiological evidence. Breast cancer Res 2008:10(1):201.

29. Kolb T.M., Lichy J., Newhouse J.H. Comparison of the performance of screening mammography, physical examination, and breast US and evaluation of factors that influence them: an analysis of 27,825 patient evaluations. Radiology 2002:225(1):165-175.

30. Willett W, Rockhill B, Hankinson S, Hunter D, Colditz G. Chapter 15: Epidemiology and Nongenetic Causes of Breast Cancer. In: Harris J, ed. Diseases of the Breast. Philadelphia: Lippincott Williams and Wilkins 2004.

31. Breast cancer and hormone replacement therapy: collaborative reanalysis of data from 51 epidemiological studies of 52,705 women with breast cancer and 108,411 women without breast cancer. Collaborative Group on Hormonal Factors in Breast Cancer. Lancet 1997;350:1047-59.

32. WHO. The International Telephone Union (ITU). A guide for countries joining the m-health program. Geneva: World Health Organization, 2012.

33. NCCN (2016) Clinical practice guidelines: Breast Cancer risk reduction, Volume 1.

34. Reiser B1. Measuring the effectiveness of diagnostic markers in the presence of measurement error through the use of ROC curves. Stat Med. 2000;19:2115-2129. [PubMed] [Google Scholar]

35. Terry, M.B.; Liao, Y.; Whittemore, A.S.; Leoce, N.; Buchsbaum, R.; Zeinomar, N.; Dite, G.S.; Chung, W.K.; Knight, J.A.; Southey, M.C.; et al. 10-year performance of four models of breast cancer risk: A validation study. Lancet Oncol. 2019, 20, 504-517.

\section{Figures}




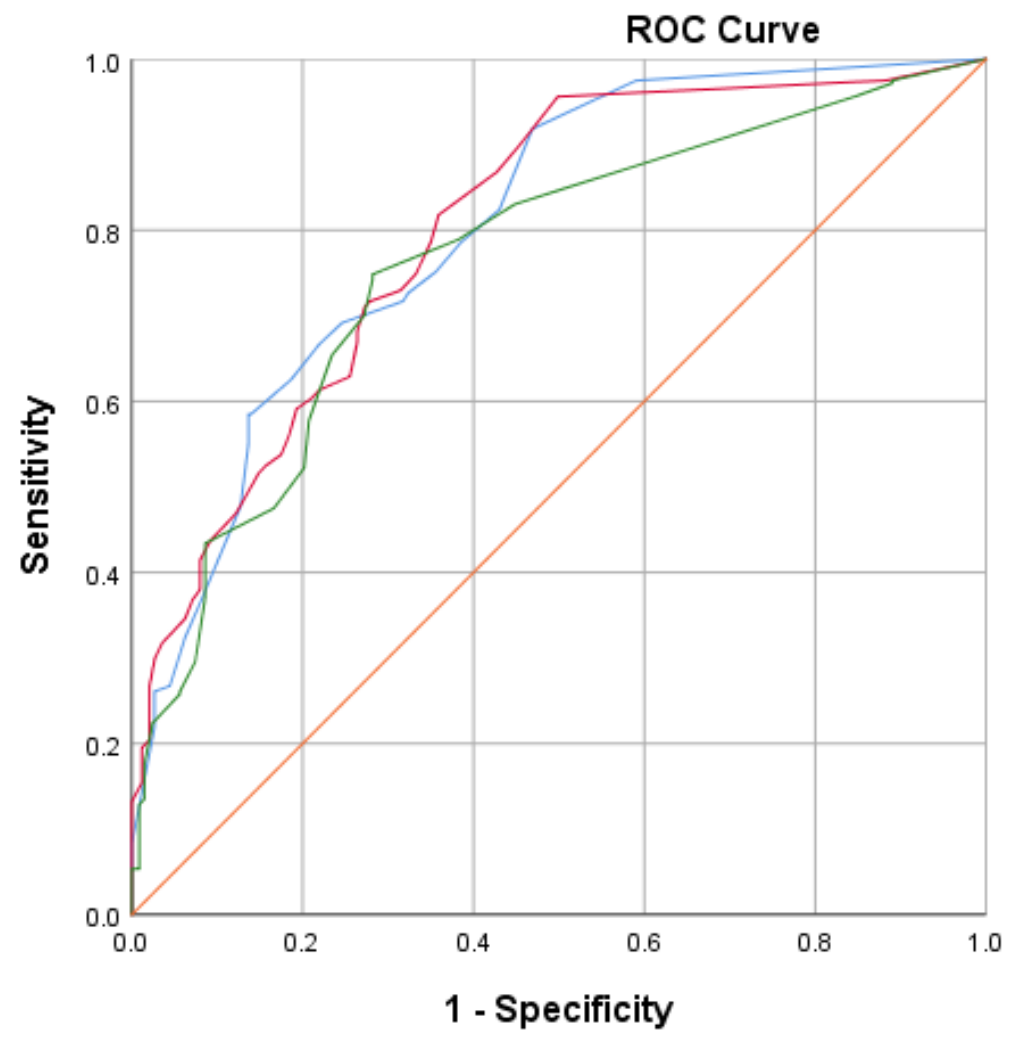

\section{Source of the Curve}

BOADIEAmodel5yearrisksore

IBIS Model 5 year risk score

- Gail Model 5year risk score

- Reference Line

Diagonal segments are produced by ties.

\section{Figure 1}

The receiver operating characteristics curve of the BOADICEA, Gail and IBIS models. This shows the discriminatory accuracy of the models. A value of 1 indicates perfect discrimination while 0.5 is by chance going to discriminate which woman will or will not have BC. 


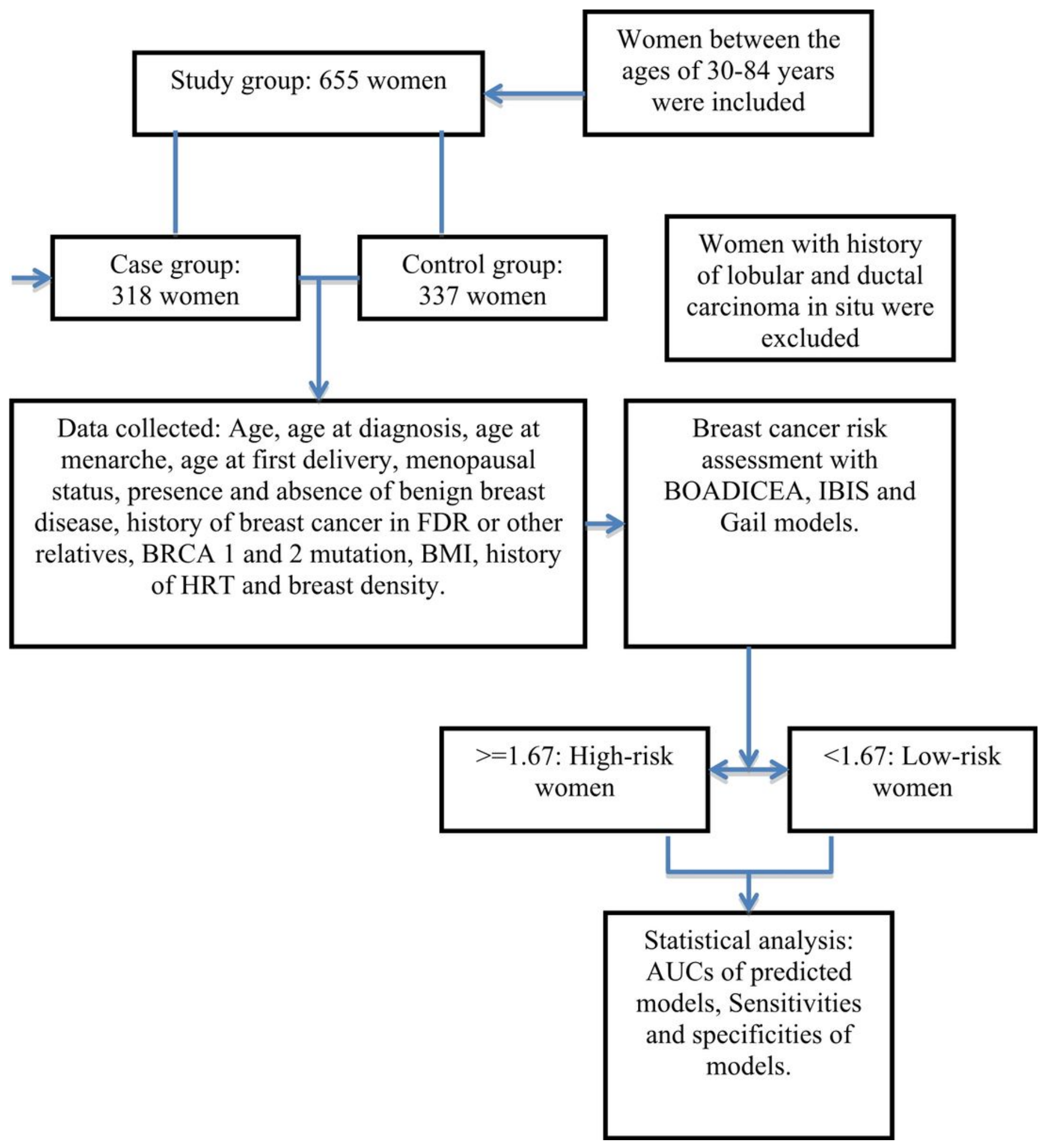

Figure 2

Flow chart of the study 